\title{
Following the primordial perturbations through a bounce with AdS/CFT correspondence
}

\author{
Lei Ming, Taifan Zheng, Yeuk-Kwan E. Cheung ${ }^{\mathrm{a}}$ \\ Department of Physics, Nanjing University, 22 Hankou Road, Nanjing 210093, China
}

Received: 22 May 2018 / Accepted: 7 September 2018 / Published online: 21 September 2018

(C) The Author(s) 2018

\begin{abstract}
A bounce universe model, known as the coupledscalar-tachyon bounce (CSTB) universe, has been shown to solve the Horizon, Flatness and Homogeneity problems as well as the Big Bang singularity problem. Furthermore a scale invariant spectrum of primordial density perturbations generated from the phase of pre-bounce contraction is shown to be stable against time evolution. In this work we study the detailed dynamics of the bounce and its imprints on the scale invariance of the spectrum. The dynamics of the gravitational interactions near the bounce point may be strongly coupled as the spatial curvature becomes big. There is no a prior reason to expect the spectral index of the primordial perturbations of matter density can be preserved. By encoding the bounce dynamics holographically onto the dynamics of dual Yang-Mills system while the latter is weakly coupled, via the AdS/CFT correspondence, we can safely evolve the spectrum of the cosmic perturbations with full control. In this way we can compare the post-bounce spectrum with the pre-bounce one: in the CSTB model we explicitly show that the spectrum of primordial density perturbations generated in the contraction phase preserves its stability as well as scale invariance throughout the bounce process.
\end{abstract}

\section{Introduction}

A universe with a bounce process has emerged over the last decade as a candidate theory for early universe dynamics as it can potentially produce a scale invariant spectrum of primordial density perturbations that matched up to the current CMB measurements. The key observation came from Wands when he noticed in 1998 that a scale invariant spectrum can be obtained in a phase of matter dominated contraction prior to a Big Bounce in his seminal work [1]. Although the spectrum followed from Wands's sim-

a e-mail: cheung@nju.edu.cn ple model was soon proven to be unstable [2], it opened up a new arena in which alternatives to Inflation were explored. ${ }^{1}$ Explicit model constructions ensue in the next two decades. This active research area has been well covered in many nice reviews over the years, the readers are referred to [322] for a repertoire of creative models, and further references.

In this article we shall continue our study of the coupled scalar-tachyon bounce (CSTB) universe model constructed from a Rolling Tachyon and its coupling to the adjoint Higgs fields in a system of non-BPS D-branes and anti-D-branes. In addition to the original model of Tachyon Inflation proposed, independently, by Sen $[23,24]$ and Gibbons [25] we incorporated the interactions of tachyon fields with the Higgs fields to model the bounce process [30]. The adjoint (real) Higgs fields live on the D-branes, and they encode, geometrically, the transverse separations between the two stacks of D-branes. The CSTB model has been shown to solve the Big Bang singularity, Horizon as well as Flatness problems with generic values of the free parameters [31,32]. Furthermore a scale invariant spectrum generated during the phase of contraction is shown to be stable; it has no implicit nor explicit time dependence in the power spectrum and it is thus stable against time evolution [33].

In [33] we also proposed a method to analyse early universe models - inflation or its alternatives - en masse by noticing that the time independence, $\eta^{W(v, m)}$, and scale invariance, $k^{L(v, m)}$, are determined by two relations,

$L(v, m)+3=-|(m-1) v-1|+3=0$,

and

$W(v, m)=[(m-1) v-1]+|(m-1) v-1|=0$,

$\overline{1}$ The inflation scenario, and its many models, is reviewed in $[26,27]$ in light of the Planck data [28,29]. 
Fig. 1 The shaded region includes all time-independent solutions satisfying $(m-1) v-1<0$ whose boundaries are defined by $(m-1) v-1=0$ delineated by thin dash lines. The purple dot-dash lines obeying $(m-1) v=-2$ represent scale-invariant as well as time-independent solutions. Another set of scale invariant solutions given by $(m-1) v=4$ (violet solid lines) have Fourier modes varying with time and therefore are not truly scale-invariant in a physical sense

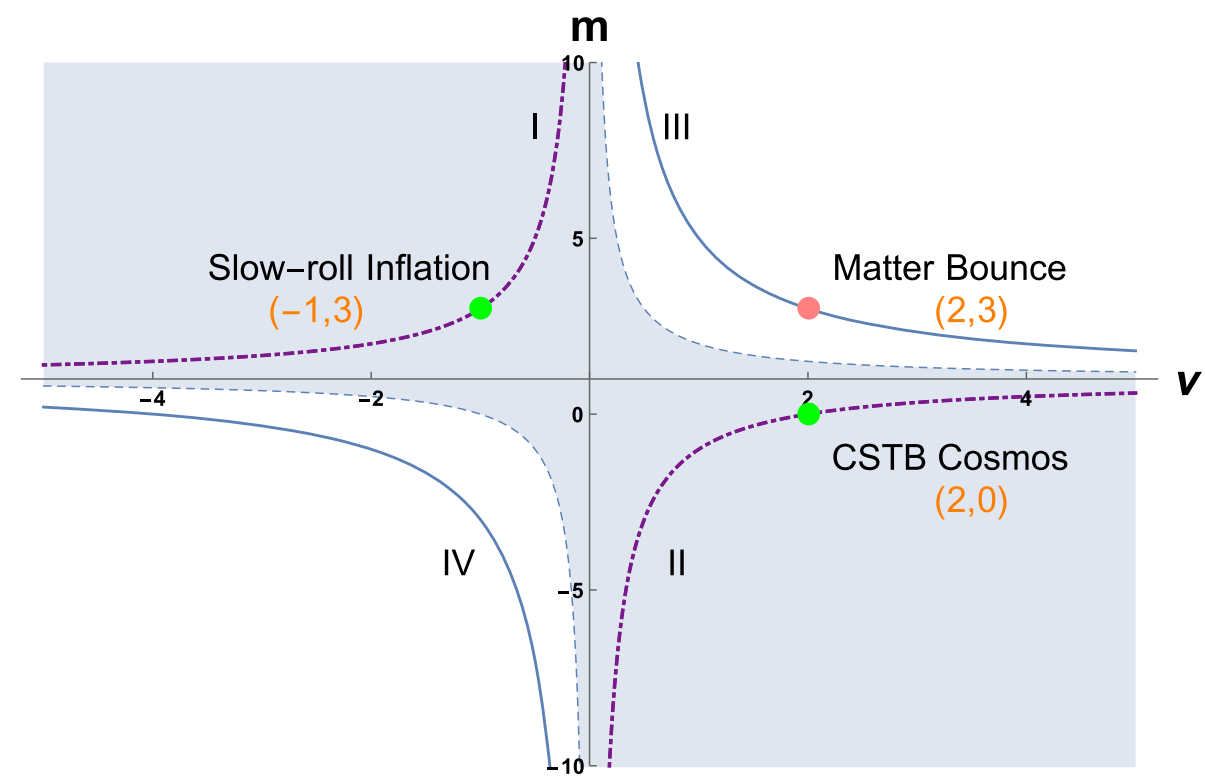

in the cosmic background evolved according to $a^{\eta}$ and the Hubble term given by $m H$. For inflation models with a canonical scalar $m=3$, with 3 coming from the spatial dimensions of our presently observable universe. In more general models $m H=3 f\left(\phi_{i}, \dot{\phi}_{i}\right) H$ with $f$ being a generic function determined by the underlying models. This method is applicable to any early universe models in which the equation of the perturbation modes, $\chi_{k}$, in k-space obeys:

$\ddot{\chi}_{k}+m H \dot{\chi}_{k}+\frac{k^{2}}{a^{2}} \chi_{k}=0, \quad a \propto \eta^{v}$,

with $m(\eta, k)$ becoming a general function of conformal time and $\mathrm{k}$-modes.

In Fig. 1 general solutions to (1) and (2) are plotted in the $(\nu, m)$ parameter space: the shaded region includes all time-independent solutions satisfying $(m-1) v-1<0$ whose boundaries are defined by $(m-1) v-1=0$ delineated by thin dash lines. The purple dot-dash lines, obeying $(m-1) v=-2$, represent scale-invariant and timeindependent solutions. Another set of scale-invariant solutions given by $(m-1) v=4$ (blue solid lines) have Fourier modes varying with time and therefore are not truly scaleinvariant in a physical sense. A few interesting solutions are marked in the figure:

$-(\nu, m)=(-1,3)$ for Slow-Roll Inflation,

$-(\nu, m)=(2,3)$ for Wands's model,

$-(v, m)=(2,0)$ for the CSTB cosmos.
The CST bounce model is an antithesis to the slow roll inflation scenario while the original matter-bounce model by Wands fails to fall on the curves of time independence. ${ }^{2}$

With the success of the primordial density generation we proceeded to obtain testable predictions from the bounce universe and their experimental verifications. In a model independent way, we studied the dark matter generation and evolutionary dynamics in a bounce universe [34,35]. We found that when dark matter coupling was of the WIMP order, dark matter was produced in plenty abundance and attained thermal equilibrium. In this case there was no constraint on the mass of dark matter produced. However when the dark matter coupling was not of the WIMP level, but much weaker instead, dark matter was produced in an out-of-thermalequilibrium route ${ }^{3}$; and thus the early universe information was encoded in the thermal evolution of the background universe as depicted in Fig. 2. Relic abundance constraint then puts dark matter mass and coupling strength on a characteristic curve [34] which could be tested in dark matter detection experiments [36].

We then proceeded to predict the number of dark matter particles detected per kilogram-year for heavy dark matter detections in nuclear-recoil experiments [37] and for light dark matter in electron-scattering experiments [38]. Together with cosmological surveys [39] we are optimistic that in the near future the bounce universe can be distinguished from inflationary scenario.

\footnotetext{
2 This was called "duality" in [33]. In this paper we reserve the use of "duality" for the strong/weak duality arisen in the AdS/CFT correspondence.

${ }^{3}$ Dark matter is produced, but the decay process of dark matter is negligible.
} 
Fig. 2 The dark matter production and evolution in time is plotted. The Y-axis is dark matter number density per entropy. A few characteristic temperatures are indicated on the time axis. When dark matter is produced in plenty abundance to reach the thermal equilibrium envelope, early universe information is washed out. When dark matter production exceed its co-annihilation rate, the observed dark matter relic density constraint then relates dark matter mass and its coupling strength [34]

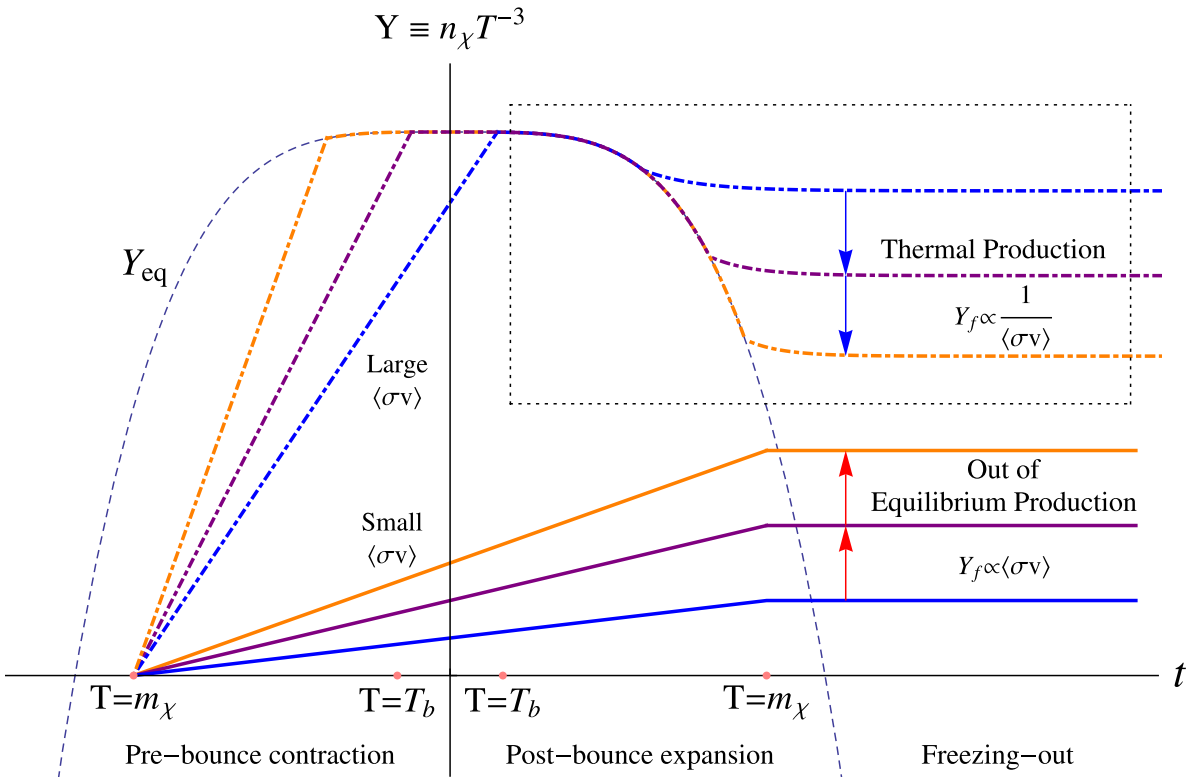

In this paper we shall focus on the evolution of the spectral index of the primordial perturbations as the CSTB cosmos goes through a bounce. We recall that the spectrum of density perturbations is generated during the matter dominated contraction, and we would like to know whether its scale invariance as well as its time dependence are altered by the bounce process, noting that at the bounce point the size of the universe reaches its minimum and the gravitational interactions may be strongly coupled. We therefore have no prior knowledge whether the bounce dynamics is under control.

To the merit of the coupled-scalar-tachyon bounce universe model, it is a model constructed by taking a consistent truncation of D-branes dynamics. It is then straightforward to embed the model in type IIB string theory and use the AdS/CFT correspondence to study the evolution of the primordial density fluctuations across the bounce point when gravity becomes strongly coupled. Since the bulk/boundary correspondence is also a strong/weak duality, it means that we can map the fluctuations onto the boundary prior to the bounce point when the bulk dynamics is well under control; and let this dynamics be monitored by the boundary gauge theory during the bounce process, when the gauge fields on the boundary are weakly coupled. After the bounce we can map back to the bulk and extract the information of the scale invariance when the gravity sector is becoming weakly coupled again. These references [73-77] inspire our work as well as many aspects of our method.

The few salient features of the CST bounce universe should be incorporated at the beginning of the study. We need to make the cosmic background time dependent with a 4-dim Friedmann-Robertson-Walker being a cross section inside the $A d S_{5}$ space. The coupled Scalar-Tachyon bounce universe avoids the violation of the null energy condition by having a spatial $S^{3}[40,41]$ and obeying the "soft bounce" condition [41]. How to set up the analysis will be covered in Sect. 2. In particular we present a time dependent dilaton solution with nonzero Ramond-Ramond charges in Type IIB string theory. We describe the cosmic background in which CSTB model can be embedded. In Sect. 3, the equations of motion governing the boundary gauge fields are solved near the bounce point and matched up at different evolutionary phases. Finally the spectrum can be studied in detail throughout the entire bounce process. In Sect. 4, we summarise and discuss the results of the investigation. We conclude with an outlook for further studies on early universe models.

\section{A time dependent dilaton solution to Type IIB supergravity}

In string theory, a Dp-brane is an object with $p$ spatial dimensions on which fundamental strings can end. Randall and Sundrum $[42,43]$ found a warped solution with two stacks of D3-branes in $(3,1)$ spacetime separated in the fifth direction in an $A d S_{5}$. The standard model fields live on the visible brane and the other hidden brane can be moved to infinite distance. Thus we can view our four dimensional bounce universe to be a D3-brane ${ }^{4}$ and embed it into a full $A d S_{5} \otimes S^{5}$ spacetime, and set up the AdS/CFT dynamics thereof.

\footnotetext{
4 To be more specific this pair of D3-branes should be an end product of non-BPS D4-branes and anti-D4-branes annihilation in Type IIB string vacuum. Lacking a dynamic description of the D-brane annihilation process for cosmological modelling we shall be restricting our attention on the resultant D3-branes themselves.
} 
To achieve our goal we incorporate and develop on many pioneering attempts of applying AdS/CFT correspondence [44] to cosmological studies [45-72]. Recently in [73] a specific recipe is provided for determining the scale dependence in the cosmological perturbations when a cosmic singularity is encountered in the bulk spacetime. We also find these works [74-77] enlightening in the course of our investigation.

The AdS/CFT correspondence is also a strong/weak duality: when the bulk fields are strongly coupled, the boundary is described by a weakly coupled field theory, and vice versa. The bulk fields have dual operators prescribed by the boundary theory. The dilaton field is related to the energy density of the gauge fields, and the gauge coupling of the boundary theory is determined by the vacuum expectation values of the dilaton. Therefore the first step is to find a time dependent solution of the dilaton equation which, in turn, determines the dynamics of gauge fields on the boundary. When the boundary gauge field theory becomes weakly coupled during the contraction, we can map the density perturbations in the bulk onto the boundary and follow their evolution through the bounce holographically using the dynamics of the gauge fields.

The bounce process in the bulk could be potentially violent or highly singular in nature. Luckily this is not the case for the CST bounce model. On the one hand it enjoys a string theoretical completion at high energy and on the other hand the CST bounce universe has a minimum radius bigger than the Planck length, $\frac{1}{a_{\min }^{2}}=\frac{8 \pi}{3} G_{N} V_{0}=\frac{4 G_{N}}{3 g_{s}}\left(\frac{m_{s}}{2 \pi}\right)^{4}$. Combining these facts one can conclude that the gauge fields on the boundary evolve smoothly as the bulk space undergoes a bounce. After the bounce, we map the evolved gauge field fluctuations - using again the AdS/CFT dictionary - back to the bulk. We can do this when the gravitational dynamics returns to a weakly coupled state. The operation described above thus allows us to compare the post-bounce spectrum with the pre-bounce spectrum. We can check whether the scale invariance of the spectrum is respected by the bounce process, or not.

To this end we need to generalise the AdS/CFT correspondence to incorporate time dependence of the model in order to study how the spectrum of primordial density perturbations, which is generated prior to the bounce in CSTB model, is evolved through the bounce to our observable universe. To this end we need to find a time dependent solution to the dilaton equation in Type IIB supergravity with nonzero Ramond-Ramond potentials [78] which allows for an $A d S_{5} \otimes S^{5}$ compactification. The action of low energy effective theory of Type IIB string is given by [79]:

$$
\begin{aligned}
S_{I I B} & =S_{N S}+S_{R}+S_{C S} \\
S_{N S} & =\frac{1}{2 \kappa_{10}^{2}} \int d^{10} x \sqrt{-g} e^{-2 \phi}\left(R+4 \partial_{\mu} \phi \partial^{\mu} \phi-\frac{1}{12}\left|H_{3}\right|^{2}\right)
\end{aligned}
$$

$$
\begin{aligned}
S_{R} & =-\frac{1}{4 \kappa_{10}^{2}} \int d^{10} x \sqrt{-g}\left(\left|F_{1}\right|^{2}+\frac{1}{3 !}\left|\widetilde{F}_{3}\right|^{2}+\frac{1}{2 \times 5 !}\left|\widetilde{F}_{5}\right|^{2}\right) \\
S_{C S} & =-\frac{1}{4 \kappa_{10}^{2}} \int C_{4} \wedge H_{3} \wedge F_{3},
\end{aligned}
$$

where the form field strengths are defined as $\widetilde{F}_{3}=F_{3}-$ $C_{0} \wedge H_{3}, \widetilde{F}_{5}=F_{5}-\frac{1}{2} C_{2} \wedge H_{3}+\frac{1}{2} B_{2} \wedge F_{3}$, and $F_{1}=d C_{0}$, $F_{3}=d C_{2}, F_{5}=d C_{4}, H_{3}=d B_{2}$. The Ramond-Ramond 5 -form fluxes, sourced by the D3-branes wrapped on the $S^{5}$, should be self-dual: $\widetilde{F}_{5}=* \widetilde{F}_{5}{ }^{5}$ The Ramond-Ramond fluxes modify the covariant constant spinor condition which allows for $A d S_{5} \otimes S^{5}$ compactification of Type IIB string. The lower forms Ramond-Ramond gauge potentials couple to lower dimensional D-branes. Dilaton $\phi$ and its dynamics are thereafter the focal point of our study.

The deformed $A d S_{5} \otimes S^{5}$ spacetime metric with which we will be working is,

$$
\begin{aligned}
d s^{2}= & \frac{L^{2}}{z^{2}}\left[-d t^{2}+a^{2}(t)\left(\frac{d r^{2}}{1-r^{2}}+r^{2}\left(d \theta^{2}+\sin ^{2} \theta d \varphi^{2}\right)\right)+d z^{2}\right] \\
& +L^{2} d \Omega_{5}^{2},
\end{aligned}
$$

where $d \Omega_{5}^{2}$ being the metric of the unit $S^{5}$, and $a(t)$ being the scale factor of the 4-dimensional closed FLRW universe and $L$ the AdS radius.

The equations of motion obtained by varying (4) are [80]:

$$
\begin{aligned}
& R_{\mu \nu}+2 \partial_{\mu} \partial_{\nu} \phi-\frac{1}{4}\left(H_{3}^{2}\right)_{\mu \nu} \\
& =e^{2 \phi}\left[\frac{1}{2}\left(F_{1}^{2}\right)_{\mu \nu}+\frac{1}{4}\left(\widetilde{F}_{3}^{2}\right)_{\mu \nu}+\frac{1}{96}\left(\widetilde{F}_{5}^{2}\right)_{\mu \nu}\right. \\
& \left.\quad-\frac{1}{4} g_{\mu \nu}\left(F_{1}^{2}+\frac{1}{6} \widetilde{F}_{3}^{2}+\frac{1}{240} \widetilde{F}_{5}^{2}\right)\right] \\
& R-4 \partial_{\mu} \phi \partial^{\mu} \phi+4 \partial_{\mu} \partial^{\mu} \phi-\frac{1}{12} H^{2}=0 \\
& * \widetilde{F}_{3} \wedge H_{3}+d * d C_{0}=0, \\
& 2 d * \widetilde{F}_{3}+H_{3} \wedge \widetilde{F}_{5}+\frac{1}{2} B_{2} \wedge d \widetilde{F}_{5}-d C_{4} \wedge H_{3}=0, \\
& d * \widetilde{F}_{5}-H_{3} \wedge F_{3}=0, \\
& -2 d\left(e^{-2 \phi} * H\right)+2 d\left(C_{0} * \widetilde{F}_{3}\right)+d C_{2} \wedge \widetilde{F}_{5} \\
& +\frac{1}{2} C_{2} \wedge d \widetilde{F}_{5}-d C_{4} \wedge d C_{2}=0,
\end{aligned}
$$

where $\mu, v=0,1 \ldots 9$ and the subscripts $p$ denote the ranks of the Ramond-Ramond fields.

We need to make some sensible assumptions as well as reasonable simplifications in order to solve this formidable array of equations. A common ansatz for self-duality $\widetilde{F}_{5}=$ $F_{5}$ is [81]:

\footnotetext{
5 The field equations derived from the action (4) should be consistent with the self-duality but do not imply it.
} 


$$
\begin{aligned}
\widetilde{F}_{5}= & r\left(\sqrt{-\operatorname{det} g_{\alpha \beta}} d x^{0} \wedge d x^{1} \wedge d x^{2} \wedge d x^{3} \wedge d x^{4}\right. \\
& \left.-\sqrt{\operatorname{det} g_{a b}} d x^{5} \wedge d x^{6} \wedge d x^{7} \wedge d x^{8} \wedge d x^{9}\right),
\end{aligned}
$$

with $r$ being a constant related to the total fluxes piercing through the $S^{5}$. Coordinates on $A d S_{5}$ and $S^{5}$ are labelled by $\alpha, \beta=0, \ldots 4$ and $a, b=5, \ldots 9$ respectively. Recalling that $\widetilde{F}_{5}=d C_{4}-\frac{1}{2} C_{2} \wedge d B_{2}+\frac{1}{2} B_{2} \wedge d C_{2}$, we can further assume that $B_{2}$ and $C_{2}$ live in the $A d S_{5}$ and $d C_{4}$ lives in the $S^{5}$. In the orthonormal basis we express them as:

$$
\begin{aligned}
& B_{2}=f_{1} d y^{0} \wedge d y^{i}+f_{2} d y^{i} \wedge d y^{j}+f_{3} d y^{i} \wedge d y^{4}+f_{4} d y^{0} \wedge d y^{4} \\
& C_{2}=g_{1} d y^{0} \wedge d y^{i}+g_{2} d y^{i} \wedge d y^{j}+g_{3} d y^{i} \wedge d y^{4}+g_{4} d y^{0} \wedge d y^{4}
\end{aligned}
$$

where $i=1,2,3,\left\{d y^{\mu}\right\}$ are the orthonormal basis, i.e. $d y^{\mu}=\sqrt{g_{\mu \mu}} d x^{\mu}$.

To ameliorate difficulty caused by the form fields we assume that the coefficients $f_{1} \ldots, g_{1} \ldots$ are at most linear in $y^{0}$ and $y^{4}$. Then the $A d S_{5}$ part of $\widetilde{F}_{5}$ :

$$
\begin{aligned}
\frac{1}{2}\left(B_{2} \wedge d C_{2}-C_{2} \wedge d B_{2}\right)= & \frac{3}{2}\left[f_{1} \frac{\partial g_{2}}{\partial y^{4}}+f_{3} \frac{\partial g_{2}}{\partial y^{0}}+f_{2}\left(\frac{\partial g_{1}}{\partial y^{4}}+\frac{\partial g_{3}}{\partial y^{0}}\right)\right. \\
& \left.-g_{1} \frac{\partial f_{2}}{\partial y^{4}}-g_{3} \frac{\partial f_{2}}{\partial y^{0}}-g_{2}\left(\frac{\partial f_{1}}{\partial y^{4}}+\frac{\partial f_{3}}{\partial y^{0}} B i g g\right)\right] .
\end{aligned}
$$

We will henceforth take these $f_{i}$ to be constants and $g_{j}$ to be linear in $y^{0}$ and $y^{4}$. The constant, $r$, mentioned above (12) becomes:

$r=\frac{3}{2}\left(f_{1} h_{3}+f_{3} h_{2}+f_{2} h_{1}\right)$,

where $h_{1}=\frac{\partial g_{1}}{\partial y^{4}}+\frac{\partial g_{3}}{\partial y^{0}}, h_{2}=\frac{\partial g_{2}}{\partial y^{0}}$ and $h_{3}=\frac{\partial g_{2}}{\partial y^{4}}$. Since $f_{4}$ and $g_{4}$ do not appear in the equations of the form fields, we will take them to be zero. Thus we have,

$$
\begin{aligned}
H_{3}= & d B_{2}=0, \\
d C_{2}= & h_{1} d y^{0} \wedge d y^{i} \wedge d y^{4}+h_{2} d y^{0} \wedge d y^{i} \wedge d y^{j} \\
& +h_{3} d y^{i} \wedge d y^{j} \wedge d y^{4}, \\
d C_{4}= & -r d y^{5} \wedge d y^{6} \wedge d y^{7} \wedge d y^{8} \wedge d y^{9} .
\end{aligned}
$$

Putting these ansatz of the tensor fields into Eqs. (8) to (11) we arrive at

$$
\begin{aligned}
\frac{\partial C_{0}}{\partial y^{4}} & =-r \frac{h_{3}}{h_{1}}, \\
\frac{\partial C_{0}}{\partial y^{0}} & =-r \frac{h_{2}}{h_{1}}, \\
h_{1}^{2} & =h_{2}^{2}-h_{3}^{2} .
\end{aligned}
$$

Likewise it is obvious that we should endow the axion field $C_{0}$ linear dependence in $y^{0}$ and the spatial direction, $y^{4}$, transverse to our 4-dimensional universe inside the $A d S_{5}$, to be consistent with the homogeneous and isotropic nature of the 4-dim FRW spacetime. So far we have set up the ansatz for the form fields with two free coefficient functions $f_{i}$ and $h_{j}$.

At this point we are ready to tackle (7), the Euler-Lagrange equation of $\phi$ :

$2 \Delta_{\mu} \partial_{\nu} \phi=4 \partial_{\mu} \phi \partial_{\nu} \phi-\frac{1}{2} g_{\mu \nu}\left(R+4 g^{\lambda \rho} \partial_{\lambda} \phi \partial_{\rho} \phi\right)$.

Putting Eqs. (20) to (23) into (6), together with the metric (5), we obtain the equations of $\phi$ when $\mu \nu=00, i i, 44$,

$$
\begin{aligned}
& \frac{3\left(\dot{a}^{2}+1\right)}{a^{2}}-\frac{6}{z^{2}}+2 \dot{\phi}^{2}+2 \phi_{, z}^{2}+\frac{6}{a^{2}} \phi_{, i}^{2} \\
& =e^{2 \phi} \frac{L^{2}}{z^{2}}\left(\frac{r^{2} h_{2}^{2}}{2 h_{1}^{2}}-3 h_{1}^{2}-3 h_{2}^{2}+\frac{9}{2} h_{3}^{2}\right), \\
& \frac{6 a^{2}}{z^{2}}-2 a \ddot{a}-\dot{a}^{2}-1+2 a^{2} \dot{\phi}^{2}-2 a^{2} \phi_{, z}^{2}-2 \phi_{, i}^{2} \\
& =e^{2 \phi} \frac{a^{2} L^{2}}{z^{2}} \frac{15 h_{1}^{2}}{2}, \\
& \frac{6}{z^{2}}-\frac{3 \ddot{a}}{a}-\frac{3\left(\dot{a}^{2}+1\right)}{a^{2}}+2 \dot{\phi}^{2}+2 \phi_{, z}^{2}-\frac{6}{a^{2}} \phi_{, i}^{2} \\
& =e^{2 \phi} \frac{L^{2}}{z^{2}}\left(\frac{r^{2} h_{3}^{2}}{2 h_{1}^{2}}+3 h_{1}^{2}+\frac{9}{2} h_{2}^{2}-3 h_{3}^{2}\right),
\end{aligned}
$$

where $\dot{\phi}=\frac{\partial \phi}{\partial t}, \phi_{, z}=\frac{\partial \phi}{\partial z}$ and $\phi_{, i}=\frac{\partial \phi}{\partial y^{i}}$.

These are quadratic first-order partial differential equations of $\phi$. Usually they are also hard to solve. If we view them as linear equations of $\dot{\phi}^{2}, \phi_{, z}^{2}$ and $\phi_{, i}^{2}$, life becomes easier:

$$
\begin{aligned}
\dot{\phi}^{2}= & \frac{1}{4} e^{2 \phi} \frac{L^{2}}{z^{2}}\left(\frac{r^{2} h_{2}^{2}}{3 h_{1}^{2}}+\frac{r^{2} h_{3}^{2}}{6 h_{1}^{2}}+\frac{13}{2} h_{1}^{2}-\frac{1}{2} h_{2}^{2}+2 h_{3}^{2}\right) \\
& +\frac{3 \ddot{a}}{4 a}-\frac{1}{z^{2}} \\
\frac{2 \phi_{, i}^{2}}{a^{2}}= & \frac{1}{6}\left[e^{2 \phi} \frac{L^{2}}{z^{2}}\left(\frac{r^{2}}{2}-\frac{27}{2} h_{1}^{2}\right)+\frac{12}{z^{2}}\right. \\
& \left.-\frac{6\left(\dot{a}^{2}+1\right)}{a^{2}}-\frac{3 \ddot{a}}{a}\right] \\
\phi_{, z}^{2}= & \frac{1}{4} e^{2 \phi} \frac{L^{2}}{z^{2}}\left(\frac{r^{2} h_{2}^{2}}{6 h_{1}^{2}}+\frac{r^{2} h_{3}^{2}}{3 h_{1}^{2}}\right. \\
& \left.-\frac{13}{2} h_{1}^{2}+2 h_{2}^{2}-\frac{1}{2} h_{3}^{2}\right)+\frac{1}{z^{2}}
\end{aligned}
$$

Compatible with homogeneity and isotropy, we let $\phi$ to be spatially homogeneous. The right side of equation (28) being zero implies that 
$e^{2 \phi} \frac{L^{2}}{z^{2}}=\left(\frac{6\left(\dot{a}^{2}+1\right)}{a^{2}}+\frac{3 \ddot{a}}{a}-\frac{12}{z^{2}}\right)\left(\frac{r^{2}}{2}-\frac{27}{2} h_{1}^{2}\right)^{-1}$.

Substituting this back into (27) we obtain

$\dot{\phi}=\frac{1}{2} \sqrt{\frac{6 m\left(\dot{a}^{2}+1\right)}{a^{2}}+\frac{3(m+1) \ddot{a}}{a}-\frac{12 m+4}{z^{2}}}$,

with

$m=\left(\frac{r^{2}}{3}+\frac{r^{2} h_{3}^{2}}{2 h_{1}^{2}}+\frac{3}{2} h_{1}^{2}+\frac{9}{2} h_{2}^{2}-3 h_{3}^{2}\right)\left(\frac{r^{2}}{2}-\frac{27}{2} h_{1}^{2}\right)^{-1}$.

This constant encodes the effects of form fields $C_{2}, B_{2}, C_{0}$ in the dynamics equation of the dilaton, $\phi$.

At this point we have set up a consistent flux compactification of Type IIB string theory on $A d S_{5} \otimes S^{5}$ with a proper time dependence in the 4-dimensional FRW subspace of the $A d S_{5}$. This is thus a starting point for utilising AdS/CFT correspondence in a cosmological study. The D3-brane on which our universe resides is viewed as a constant $z$ slice of the $A d S_{5}$, and its evolution is denoted by the scale factor $a$. In Sect. 3, we are going to utilise AdS/CFT correspondence to study the scale invariance of the primordial spectrum of density perturbations throughout the bounce process.

\section{The evolution of the gauge-field fluctuations}

The analysis in this section closely follow the general techniques provided by [73] with a few marked differences which will be explained below in detail. We focus on normalisable bulk modes since we are interested in the evolution of the linearised bulk fluctuations. Note that in this project we are interested in the primordial matter density perturbations generated by the quantum fluctuations of the scalar field. In the CSTB model this role is played by the tachyon field. ${ }^{6}$ The scale dependence of this primordial spectrum can be tested against the current $\mathrm{CMB}$ measurements with extreme high precision.

Let us emphacise that we do not take this scalar in the bulk to be the dilaton field like it was done in [73]. The detailed analysis goes through as the mathematical framework allows to use the equations of motion to evolve the boundary theory. Using the AdS/CFT correspondence we can evolve the corresponding linear fluctuation of the gauge field $A_{\mu}$ on the boundary of $A d S_{5}$. The initial conditions for the gauge fields on the boundary are set by the primordial fluctuations of the scalar fields via the correspondence; and thus the evolution

\footnotetext{
${ }^{6}$ The effective mass of the scalar field representing the separation of the stack of non-BPS D4-branes and anti-D4-branes is much heavier, it thus describes the evolution of the underlying cosmos [33].
}

of the gauge fields is thus well-defined at all time and under control. The primordial curvature perturbations in the CSTB model are related to the spectrum of tachyon matter perturbations by a constant factor in the long wavelength limit [33]. The curvature perturbations in 5-dimensional bulk can also be studied within this framework. In [74] it was shown that a generalisation of the uniform curvature gauge to the 5dimensional case allows one to relate the curvature perturbations to scalar field perturbations, as usual. A straightforward calculation shows that this is also the case in our model: to the order of interest, the calculations performed on the scalar field can be related to the curvature perturbations in the longitudinal gauge.

The boundary gauge theory is described by $\mathcal{N}=4$ SYM theory, the gauge coupling of which is determined by the dilaton, $g_{Y M}^{2}=e^{\phi}$. Thus at linear level the effect of dilaton on the gauge field fluctuations will be its time-dependence, which we have analysed in detail in Sect. 2. We shall now turn our attention to the asymptotical properties of gauge coupling in order to check the applicability of AdS/CFT correspondence to our bounce universe.

\subsection{The scale factor of CSTB and the applicability of AdS/CFT}

Recall that in the CSTB model, the universe undergoes a contraction phase, deflation, the bounce, at which a phase of "locked inflation" takes place when the tachyon is locked at the peak of potential hill. As the universe inflates, the oscillations of the Higgs fields get red-shifted and eventually fails to lock the tachyon at the peak of its potential. As the tachyon rolls down its potential, it becomes a normal form of matter with no pressure [23]. The cosmic evolution is schematically depicted in Fig. 3.

In the subsequent calculations we are going to take three different, but constant, values of the Hubble parameters [30, $31]$,

$$
\begin{aligned}
& \text { Contraction : } a=a_{2}(-H t)^{\frac{2}{3}}, t \leq-t_{2}, \\
& \text { Deflation : } a=a_{1} e^{-H t},-t_{2}<t<-t_{1}, \\
& \text { Smoothbounce : } a=a_{0} \cosh (H t),-t_{1} \leq t \leq t_{1}, \\
& \text { Lockedinflation }: a=a_{1} e^{H t}, t_{1}<t<t_{2}, \\
& \text { Expansion : } a=a_{2}(H t)^{\frac{2}{3}}, t \geq t_{2},
\end{aligned}
$$

where $t_{1}$ is the time when locked inflation starts, $t_{2}$ is when it ends, $H$ is the Hubble constant during inflation and $a_{0}$ is the minimal radius of the universe. The bounce process is symmetric about $t=0 .^{7}$

\footnotetext{
$\overline{7}$ In the absence of entropy creation the bounce process is symmetric about $t=0$. Considering entropy creation is outside the scope of this paper and it does not affect the analysis undertaken in this work.
} 


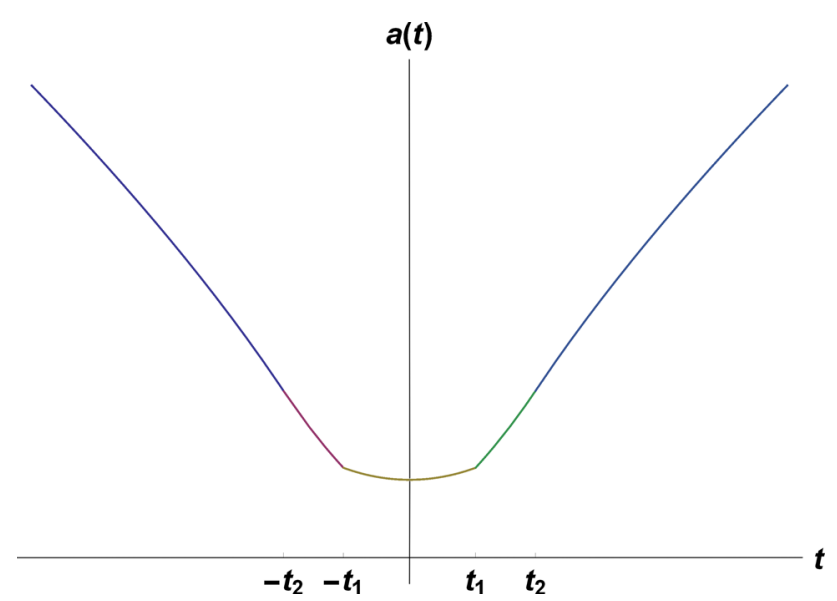

Fig. 3 The CSTB universe undergoes five phases: Tachyon matter dominated contraction, deflation, smooth bounce and locked inflation at the peak of the tachyon potential when the tachyon is locked by the fast oscillations of the Higgs field. After that a period of tachyon-matterdominated rolling expansion ensues.

Now let us check whether the correspondence is applicable at early and late times in our CSTB universe, insert (36) into (30) we obtain

$e^{2 \phi}=\frac{24-4 z^{2}\left[t^{-2}+3 a_{2}^{-2}(H t)^{-\frac{4}{3}}\right]}{L^{2}\left(27 h_{1}^{2}-r^{2}\right)}$,

the limit of 't Hooft coupling, $\lambda=g_{s} N=e^{\phi} N$, would be $\frac{N}{L} \sqrt{\frac{24}{27 h_{1}^{2}-r^{2}}}$ when $|t| \rightarrow \infty$. The framework we set up in Sect. 2 is fairly free: in the large $N$ limit we have an alternative ansatz to choose for $h_{i}$ and $f_{j}$, provided that $27 h_{1}^{2}-r^{2}$ is positive and large enough. In this case the string coupling $g_{s}=e^{\phi}$ is small and has a bound at all times, which in turn makes the 't Hooft coupling $\lambda=g_{s} N$ decrease as $|t|$ decrease. In other words the supergravity bulk theory will become less and less valid when the universe contracts. In the ensuing deflation phase (35), we have

$e^{2 \phi}=\frac{24-2 z^{2}\left(9 H^{2}+6 a_{1}^{-2} e^{-2 H t}\right)}{L^{2}\left(27 h_{1}^{2}-r^{2}\right)}$.

Similarly we can see that the gauge coupling $g_{Y M}$ is small and decreases with $|t|$ decreasing, it is certainly bounded when $t_{1}<|t|<t_{2}$. Therefore the boundary gauge theory will become more and more valid as the universe contracts.

During the smooth bounce process (34) the square of coupling is

$e^{2 \phi}=\frac{24-18(H z)^{2}}{L^{2}\left(27 h_{1}^{2}-r^{2}\right)}$,

this is independent of time. As discussed above, this choice of parameters can give us a small coupling constant $g_{Y M}^{2}$. We note, however, that we may have to consider the effect of $z$. It cannot be too far from the boundary $z=0$, for instance if $\frac{N}{L} \ll \sqrt{27 h_{1}^{2}-r^{2}}$ then $z$ has to be $H z \sim \mathcal{O}(1)$. In this case the Yang-Mills coupling and the 't Hooft coupling are both small so the boundary gauge theory is well behaved around the bounce point. All in all the AdS/CFT correspondence with appropriate choice of parameters is an appropriate tool for studying the physics around the bounce point.

\subsection{An overview of the physics analysis around the bounce point}

As we shall see below we are going to map the bulk fluctuations onto the boundary. We would like to choose the mapping point $\pm t_{m}$ to be when $g_{s} N \sim \mathcal{O}(1)$, where the gauge theory becomes weakly coupled and the gravity theory strongly coupled. And $\left|t_{m}\right|$ should be a little large than $\left|t_{2}\right|$, which means the mapping happens during contraction phase and is close to deflation. Besides, in the correspondence language $t_{1}$ should be the timing when the coupling decreases to and stays as a small enough constant.

The boundary theory is strongly coupled in the far past. As the universe contracts, the bulk gravity theory becomes more and more strongly coupled. Long before approaching the bounce point, we map the fluctuations onto the boundary where it is weakly coupled. We monitor the evolution of the gauge fields well after the bounce ends when the bulk returns to a weakly coupled state. In this and the following subsections we are going to use the notations in [73] and review their setup for the equation of motion for the gauge fields.

First making a gauge choice $A_{0}=0$ and imposing an additional constraint $\partial^{i} A_{i}=0$ so that the Gauss Law constraint is automatically solved. By rescaling the gauge fields, $A_{\mu} \rightarrow \widetilde{A}_{\mu} \equiv e^{-\phi / 2} A_{\mu}$, an effective mass,

$M_{Y M}^{2}=\frac{\ddot{\phi}}{2}-\frac{\dot{\phi}^{2}}{4}$,

appears in the equations of motion for the rescaled gauge fields, $\widetilde{A}_{i}$,

$-\partial^{\mu} \partial_{\mu} \widetilde{A}_{i}+M_{Y M}^{2} \widetilde{A}_{i}=0$

Upon Fourier transformation,

$\widetilde{A}_{v}\left(\xi^{\mu}\right)=\int_{-\infty}^{\infty} d^{3} \mathbf{k} c_{A}(\mathbf{k}) \widetilde{A}_{k}(t) \epsilon_{v} e^{i \mathbf{k} \cdot \xi}$,

where $\epsilon_{v}$ is the unit polarization vector. The equations of motion for the Fourier modes of the gauge fields $\widetilde{A}$ become [63]:

$\ddot{\widetilde{A}}_{k}+\left(k^{2}+M_{Y M}^{2}\right) \widetilde{A}_{k}=0$. 
To summarise: using the results in Sect. 2, we obtain the scale factor, $a$, and the corresponding coupling constant, $g_{s}=g_{Y M}^{2}=e^{\phi}$, in the five cosmic epochs of the CSTB universe. In this section we have checked thoroughly the applicability of the AdS/CFT correspondence in the cosmological context of the CSTB model. We hence conclude that, with a suitable choice of the ansatz parameters in Sect. 2, the bulk theory can be made weakly coupled at early and late times. The boundary theory can also be made weakly coupled and well-behaved around the bounce point. This is what we have realised in the model building. We briefly review the setup for the gauge fields and their equations of motion. We are interested to find out if the gauge fields acquire extra scale dependence due to the bounce dynamics. Equipped with the solution of dilaton and the equations of motion for $\widetilde{A}_{k}$, we can evolve gauge field fluctuations at the boundary.

\subsection{The solution of $\widetilde{A}_{k}$ and its matching}

With the framework delineated above we are ready to use the AdS/CFT correspondence to study the dynamics of the CSTB model in detail - by projecting the bulk dynamics onto the boundary and evolve it using the boundary theory. The mapping happens at contraction and expansion phases straddling the period when the bulk becomes strongly coupled. We shall solve the equations of motion in each phase:

1. Contraction:

Putting (32) into (31) we obtain

$$
\begin{aligned}
\dot{\phi} & =\frac{1}{2} \sqrt{\frac{6 m}{a_{2}^{2}(H t)^{\frac{4}{3}}}+\frac{2 m-\frac{2}{3}}{t^{2}}-\frac{4(3 m+1)}{z^{2}}} \\
& \approx \frac{1}{2} \sqrt{\frac{2 m-\frac{2}{3}}{t^{2}}-\frac{4(3 m+1)}{z^{2}}}
\end{aligned}
$$

here we ignore the $t^{-\frac{4}{3}}$ term in the root since $\frac{1}{a_{2}^{2}}$ is much smaller compared to $H^{2}$ at large $t$. Taking up to second order of $\frac{1}{t}$, we have

$\ddot{\phi} \approx \frac{1}{2} \sqrt{-\frac{4(3 m+1)}{z^{2}}}\left[\frac{2 m-\frac{2}{3}}{4(3 m+1)} \frac{z^{2}}{t^{3}}+\frac{1}{2}\left(\frac{2 m-\frac{2}{3}}{4(3 m+1)}\right)^{2} \frac{z^{4}}{t^{5}}\right] \approx 0$,

and

$$
M_{Y M}^{2}=-\frac{1}{16}\left(\frac{2 m-\frac{2}{3}}{t^{2}}-\frac{4(3 m+1)}{z^{2}}\right) \equiv \frac{S}{t^{2}}+T,
$$

where we donate the constants as $S \equiv \frac{\frac{2}{3}-2 m}{16}$ and $T \equiv$ $\frac{3 m+1}{4 z^{2}}$. Putting it into (43) yields

$$
\widetilde{A}_{k}=L_{1}(k) \sqrt{-\beta_{1} t} J_{\sigma}\left(-\beta_{1} t\right)+L_{2}(k) \sqrt{-\beta_{1} t} N_{\sigma}\left(-\beta_{1} t\right),
$$

here $J_{\sigma}(t)$ and $N_{\sigma}(t)$ are two kinds of Bessel functions of order $\sigma \equiv \sqrt{\frac{1}{4}-S}$ and $\beta_{1} \equiv \sqrt{k^{2}+T}, L_{1,2}(k)$ are functions of $k$ and the notations are similar in the following.

2. Deflation: Putting (33) and (31) into (40) we arrive at

$$
\begin{aligned}
M_{Y M}^{2}= & \frac{3 m H e^{2 H t}}{2 a_{1}^{2} \sqrt{(9 m+3) H^{2}+\frac{6 m e^{2 H t}}{a_{1}^{2}}-\frac{12 m+4}{z^{2}}}}-\frac{3}{16}(3 m+1) H^{2} \\
& +\frac{3 m+1}{4 z^{2}}-\frac{3 m e^{2 H t}}{8 a_{1}^{2}} .
\end{aligned}
$$

In (48) near the matching point $t_{1}$, which is small, all the terms are effectively constant, we denote it as $M$. Putting it into (43) yields

$\widetilde{A}_{k}=D_{1}(k) e^{\beta_{2} t}+D_{2}(k) e^{-\beta_{2} t}$,

where $\beta_{2} \equiv \sqrt{-k^{2}-M}, M \equiv \frac{3 m H}{2 a_{1}^{2} \sqrt{(9 m+3) H^{2}+\frac{6 m}{a_{1}^{2}}-\frac{12 m+4}{z^{2}}}}$ $-\frac{3}{16}(3 m+1) H^{2}+\frac{3 m+1}{4 z^{2}}-\frac{3 m}{8 a_{1}^{2}}$.

3. The smooth bounce: Taking the first order of $t$ we obtain

$$
\begin{aligned}
M_{Y M}^{2} & =\frac{3 m H^{4} t}{\sqrt{-\frac{12 m+4}{z^{2}}+3(m+1) H^{2}+\frac{6 m}{a_{0}^{2}}}} \\
& -\frac{1}{4}\left(-\frac{3 m+1}{z^{2}}+\frac{3}{4}(m+1) H^{2}+\frac{6 m}{a_{0}^{2}}\right) \\
& \equiv P t+Q,
\end{aligned}
$$

here $P \equiv \frac{3 m H^{4}}{\sqrt{-\frac{12 m+4}{z^{2}}+3(m+1) H^{2}+\frac{6 m}{a_{0}^{2}}}}$ and $Q \equiv-\frac{1}{4}\left(-\frac{3 m+1}{z^{2}}\right.$ $\left.+\frac{3}{4}(m+1) H^{2}+\frac{6 m}{a_{0}^{2}}\right)$ are constants, which yields

$$
\begin{aligned}
\widetilde{A}_{k}= & E_{1}(k) \mathrm{Ai}\left[\frac{-k^{2}-Q-P t}{(-P)^{\frac{2}{3}}}\right] \\
& +E_{2}(k) \mathrm{Bi}\left[\frac{-k^{2}-Q-P t}{(-P)^{\frac{2}{3}}}\right],
\end{aligned}
$$

here $A i[t]$ and $B i[t]$ are Airy functions of $t$.

4. Locked inflation: In this case everything is same as deflation except the value of $t$. Therefore

$\widetilde{A}_{k}=F_{1}(k) e^{\beta_{2} t}+F_{2}(k) e^{-\beta_{2} t}$. 
5. Expansion: In this case everything is same as contraction except the value of $t$. Therefore

$$
\widetilde{A}_{k}=R_{1}(k) \sqrt{\beta_{1} t} J_{\sigma}\left(\beta_{1} t\right)+R_{2}(k) \sqrt{\beta_{1} t} N_{\sigma}\left(\beta_{1} t\right),
$$

where we re-iterate that $\{D, E, F, R\}_{1,2}(k)$ are not constant coefficients but are assumed to be functions of $k$.

We take the asymptotic expansion of large $t$ for Bessel functions so that:

$L_{1}(k) \sqrt{-\beta_{1} t} J_{\sigma}\left(-\beta_{1} t\right)=L_{1}(k) \sqrt{\frac{2}{\pi}} \cos \left(-\beta_{1} t-\frac{1+2 \sigma}{4} \pi\right)$,

$L_{2}(k) \sqrt{-\beta_{1} t} N_{\sigma}\left(-\beta_{1} t\right)=L_{2}(k) \sqrt{\frac{2}{\pi}} \sin \left(-\beta_{1} t-\frac{1+2 \sigma}{4} \pi\right)$.

Matching $\widetilde{A}_{k}$ and its derivative at $t=-t_{2}$ :

$$
\begin{aligned}
& L_{1}(k) \sqrt{\frac{2}{\pi}} \cos \left(\beta_{1} t_{2}-\frac{1+2 \sigma}{4} \pi\right)+L_{2}(k) \sqrt{\frac{2}{\pi}} \sin \left(\beta_{1} t_{2}-\frac{1+2 \sigma}{4} \pi\right) \\
& =D_{1}(k) e^{-\beta_{2} t_{2}}+D_{2}(k) e^{\beta_{2} t_{2}} \\
& \sqrt{\frac{2}{\pi}} \frac{\beta_{1}}{\beta_{2}}\left[-L_{1}(k) \sin \left(\beta_{1} t_{2}-\frac{1+2 \sigma}{4} \pi\right)+L_{2}(k) \cos \left(\beta_{1} t_{2}-\frac{1+2 \sigma}{4} \pi\right)\right] \\
& =D_{1}(k) e^{-\beta_{2} t_{2}}-D_{2}(k) e^{\beta_{2} t_{2}},
\end{aligned}
$$

which yields

$$
\begin{aligned}
D_{1}(k)= & \frac{2}{\pi} \frac{e^{-\beta_{2} t_{2}}}{2 \beta_{2}}\left\{\left[\beta_{2} \cos \left(\beta_{2} t_{2}-\frac{1+2 \sigma}{4} \pi\right)\right.\right. \\
& \left.-\beta_{1} \sin \left(\beta_{2} t_{2}-\frac{1+2 \sigma}{4} \pi\right)\right] L_{1}(k) \\
& +\left[\beta_{1} \cos \left(\beta_{2} t_{2}-\frac{1+2 \sigma}{4} \pi\right)\right. \\
& \left.\left.+\beta_{2} \sin \left(\beta_{2} t_{2}-\frac{1+2 \sigma}{4} \pi\right)\right] L_{2}(k)\right\},
\end{aligned}
$$

and

$$
\begin{aligned}
D_{2}(k)= & \frac{2}{\pi} \frac{e^{-\beta_{2} t_{2}}}{2 \beta_{2}}\left\{\left[\beta_{2} \cos \left(\beta_{2} t_{2}-\frac{1+2 \sigma}{4} \pi\right)\right.\right. \\
& \left.+\beta_{1} \sin \left(\beta_{2} t_{2}-\frac{1+2 \sigma}{4} \pi\right)\right] L_{1}(k) \\
& +\left[-\beta_{1} \cos \left(\beta_{2} t_{2}-\frac{1+2 \sigma}{4} \pi\right)\right. \\
& \left.\left.+\beta_{2} \sin \left(\beta_{2} t_{2}-\frac{1+2 \sigma}{4} \pi\right)\right] L_{2}(k)\right\} .
\end{aligned}
$$

We assume the arguments of both Airy functions to be small and that the $(-P)^{\frac{1}{3}} t$ term dominate. We can thus expand the Airy functions asymptotically to first power in $q \equiv \frac{-k^{2}-Q-P t}{(-P)^{\frac{2}{3}}}:$

$$
\begin{aligned}
& E_{1}(k) \operatorname{Ai}(q)=\frac{\left(\frac{1}{3}\right)^{\frac{2}{3}}}{\Gamma\left(\frac{2}{3}\right)} E_{1}(k), \\
& E_{2}(k) \operatorname{Bi}(q)=\left[\frac{\left(\frac{1}{3}\right)^{\frac{1}{6}}}{\Gamma\left(\frac{2}{3}\right)}+\frac{\left(\frac{1}{3}\right)^{\frac{5}{6}}}{\Gamma\left(\frac{4}{3}\right)^{2}} q\right] E_{2}(k) .
\end{aligned}
$$

Now we can match $\widetilde{A}_{k}$ and its derivatives at the end of deflation and at the beginning of inflation, which we denote as $-t_{1}$ and $t_{1}$ respectively. Matching $\widetilde{A}_{k}$ yields:

$$
\begin{aligned}
& D_{1}(k) e^{-\beta_{2} t_{1}}+D_{2}(k) e^{\beta_{2} t_{1}}=\frac{\left(\frac{1}{3}\right)^{\frac{2}{3}}}{\Gamma\left(\frac{2}{3}\right)} E_{1}(k) \\
& +\left[\frac{\left(\frac{1}{3}\right)^{\frac{1}{6}}}{\Gamma\left(\frac{2}{3}\right)}+\frac{\left(\frac{1}{3}\right)^{\frac{5}{6}}}{\Gamma\left(\frac{4}{3}\right)^{2}} q_{1}\right] E_{2}(k), \\
& F_{1}(k) e^{\beta_{2} t_{1}}+F_{2}(k) e^{-\beta_{2} t_{1}}=\left[\frac{\left(\frac{1}{3}\right)^{\frac{2}{3}}}{\Gamma\left(\frac{2}{3}\right)}+\frac{\left(\frac{1}{3}\right)^{\frac{4}{3}}}{\Gamma\left(\frac{4}{3}\right)} q_{2}\right] E_{1}(k) \\
& +\left[\frac{\left(\frac{1}{3}\right)^{\frac{1}{6}}}{\Gamma\left(\frac{2}{3}\right)}-\frac{\left(\frac{1}{3}\right)^{\frac{5}{6}}}{\Gamma\left(\frac{4}{3}\right)} q_{2}\right] E_{2}(k),
\end{aligned}
$$

where $\left.q_{1} \equiv q\right|_{t=-t_{1}}$ and $\left.q_{2} \equiv q\right|_{t=t_{1}}$.

Matching $\dot{\widetilde{A}}_{k}$ yields:

$D_{1}(k) \beta_{2} e^{-\beta_{2} t_{1}}-D_{2}(k) \beta_{2} e^{\beta_{2} t_{1}}=\frac{\left(\frac{1}{3}\right)^{\frac{5}{6}}}{\Gamma\left(\frac{4}{3}\right)}(-P)^{\frac{1}{3}} E_{2}(k)$,

and

$$
F_{1}(k) \beta_{2} e^{\beta_{2} t_{1}}-F_{2}(k) e^{-\beta_{2} t_{1}}=\frac{\left(\frac{1}{3}\right)^{\frac{4}{3}}}{\Gamma\left(\frac{4}{3}\right)} P^{\frac{1}{3}} E_{1}(k)+\frac{\left(\frac{1}{3}\right)^{\frac{5}{6}}}{\Gamma\left(\frac{4}{3}\right)}(-P)^{\frac{1}{3}} .
$$

Solving (62) to (65) we obtain the solutions to $F_{1}$ and $F_{2}$ as follows:

$$
F_{1}(k)=\frac{1}{6 \beta_{2} \Gamma\left(\frac{4}{3}\right)(-P)^{\frac{1}{3}} e^{2 \beta_{2} t_{1}}}\left[D_{1}(k) I_{1}+D_{2}(k) e^{2 \beta_{2} t_{1}} I_{2}\right],
$$

where

$$
\begin{aligned}
I_{1}= & -3^{\frac{1}{3}} \Gamma\left(\frac{2}{3}\right)(-P)^{\frac{2}{3}}+\beta_{2}(-P)^{\frac{1}{3}} \\
& \times\left[3^{\frac{1}{3}} \Gamma\left(\frac{2}{3}\right)\left(q_{1}+q_{2}\right)+9 \Gamma\left(\frac{4}{3}\right)\right] \\
& -\beta_{2}^{2}\left[3^{\frac{1}{3}} \Gamma\left(\frac{2}{3}\right) q_{1} q_{2}+3 \Gamma\left(\frac{4}{3}\right)\left(q_{1}+2 q_{2}\right)\right],
\end{aligned}
$$


and

$$
\begin{aligned}
I_{2}= & -3^{\frac{1}{3}} \Gamma\left(\frac{2}{3}\right)(-P)^{\frac{2}{3}}+\beta_{2}(-P)^{\frac{1}{3}} \\
& \times\left[-3^{\frac{1}{3}} \Gamma\left(\frac{2}{3}\right)\left(q_{1}+q_{2}\right)-3 \Gamma\left(\frac{4}{3}\right)\right] \\
& -\beta_{2}^{2}\left[3^{\frac{1}{3}} \Gamma\left(\frac{2}{3}\right) q_{1} q_{2}+3 \Gamma\left(\frac{4}{3}\right)\left(q_{1}+2 q_{2}\right)\right] ;
\end{aligned}
$$

while

$$
F_{2}(k)=\frac{1}{6 \beta_{2} \Gamma\left(\frac{4}{3}\right)(-P)^{\frac{1}{3}}}\left[-D_{1}(k) J_{1}+D_{2}(k) e^{2 \beta_{2} t_{1}} J_{2}\right],
$$

with $J_{1}$ and $J_{2}$ given by

$$
\begin{aligned}
J_{1}= & -3^{\frac{1}{3}} \Gamma\left(\frac{2}{3}\right)(-P)^{\frac{2}{3}}+\beta_{2}(-P)^{\frac{1}{3}}\left[3^{\frac{1}{3}} \Gamma\left(\frac{2}{3}\right)\left(q_{1}-q_{2}\right)+3 \Gamma\left(\frac{4}{3}\right)\right] \\
& +\beta_{2}^{2}\left[3^{\frac{1}{3}} \Gamma\left(\frac{2}{3}\right) q_{1} q_{2}+3 \Gamma\left(\frac{4}{3}\right)\left(q_{1}+2 q_{2}\right)\right],
\end{aligned}
$$

and

$$
\begin{aligned}
J_{2}= & 3^{\frac{1}{3}} \Gamma\left(\frac{2}{3}\right)(-P)^{\frac{2}{3}}+\beta_{2}(-P)^{\frac{1}{3}}\left[3^{\frac{1}{3}} \Gamma\left(\frac{2}{3}\right)\left(q_{1}+q_{2}\right)+9 \Gamma\left(\frac{4}{3}\right)\right] \\
& -\beta_{2}^{2}\left[3^{\frac{1}{3}} \Gamma\left(\frac{2}{3}\right) q_{1} q_{2}+3 \Gamma\left(\frac{4}{3}\right)\left(q_{1}+2 q_{2}\right)\right] .
\end{aligned}
$$

By now we have matched the solutions of $\widetilde{A}_{k}$ at $t=-t_{2}$ and $t= \pm t_{1}$; similar calculations take place at $t=t_{2}$. In this way, we obtain a full chain of relations from $L_{1,2}(k)$ to $R_{1,2}(k)$, which encodes all the information concerning the scale dependence in the power spectrum. It is then straightforward to judge if any of these functions pick up extra $k$ dependence due to the bounce.

In particular we are interested in small wavenumber limit, $k t \ll 1$, when the modes of perturbations are outside the Hubble horizon. Therefore, we have,

$\beta_{1}=\sqrt{k^{2}+T}=\sqrt{k^{2}+\frac{4(3 m+1)}{z^{2}}} \approx \sqrt{T}$,

and

$\beta_{2}=\sqrt{-k^{2}-M} \approx \sqrt{-M}$.

In addition, $Q \sim M$, we have

$q_{1}=\frac{-k^{2}-Q+P t_{1}}{(-P)^{\frac{2}{3}}} \approx \frac{-Q+P t_{1}}{(-P)^{\frac{2}{3}}}$.

Similar argument goes for $q_{2}$. Henceforth $\beta_{1,2}$ and $q_{1,2}$ are independent of $k$, with $e^{\beta_{2} t}, I_{1,2}$ and $J_{1,2}$ being independent of $k$ when $k t_{1,2} \ll 1$.

All in all one sees plainly that no extra $k$ dependence is introduced into the above relations by the bounce process: the spectral index is not altered by the bounce upon comparing the $k$ dependence in $L_{1,2}(k)$ and $R_{1,2}(k)$. To conclude: the scale invariance of the primordial density perturbations, if exists prior to the bounce, will hold throughout the bounce process in the CSTB bounce universe as it cannot be affected by the bounce dynamics.

A few comments on using AdS/CFT correspondence to reconstruct of the spectrum from boundary data are made here. As mentioned in the beginning of this section, the position of our universe brane cannot be too far away from the boundary. The field at a bulk point is reconstructed by integrating the boundary data against the boundary-to-bulk propagator, and the region is defined by the null geodesics connecting the bulk point to the boundary $[70,73]$. Therefore the reconstruction of the information can be done in a small region around the "mapping point" well before the gauge theory becomes strongly coupled again. The low energy effective theory used to model the bounce universe is that of the Higgs fields and the tachyon field, as well as their coupling term. Unlike other field theoretical model, it has a high energy completion as a string theory [30]: there is no singularity even as the scale factor achieves its minimal value,

$\frac{1}{a_{\min }^{2}}=\frac{8 \pi}{3} G_{N} V_{0}=\frac{4 G_{N}}{3 g_{s}}\left(\frac{m_{s}}{2 \pi}\right)^{4}$,

where $m_{s}$ is the string mass. The qualification for our discussions may lie in the choice of ansatz and the effects of different compactifications. A complete analysis is beyond the scope of this paper, to which we will return in a future work. In the current project we are mostly following (the spirit of) the recipe provided in [73] for the reconstruction of the bulk data from boundary. We can conclude within this framework the $k$-dependence of the bulk fluctuations is completely determined by the $k$-dependence of the fluctuations of the gauge fields, $\widetilde{A}_{k}(t)$, on the boundary. Thus the evolution of the gauge fluctuations will preserve the scale invariance of the primordial spectrum of density perturbations in the bulk across the bounce point in the CST bounce universe model.

\section{Conclusion and discussion}

In this paper we use the AdS/CFT correspondence to study whether the spectral index of the primordial perturbations is altered by the bounce dynamics in the Coupled-ScalarTachyon Bounce Universe model. Considering the string background of CSTB model, we first embed the four dimensional bounce universe into an $A d S_{5} \otimes S^{5}$ background, and find a time-dependent solution of dialton in type IIB supergravity. The dilaton solution is then used to solve for the dynamics of gauge fields living on the boundary of the $A d S_{5}$. We follow the gauge fields through the bounce process, as 
they are weakly coupled, and match their solutions at different phases of cosmic evolution.

The combined profile of gauge field evolution is smooth across the bounce point. The bounce process merely alters the amplitudes of different modes in the density perturbations, but it affects them in the same manner. It therefore does not alter the intrinsic $k$ dependence in the spectrum of primordial perturbations generated in the pre-bounce contraction. We are led to conclude that the scale invariance, if exists before the bounce, will hold throughout the bounce process. Nevertheless as we can see from (58), (59), (66) and (67), as $k$ becomes larger and larger extra $k$ dependence begins to creep out in the spectral index, the implications of which are under investigation.

No prior dilaton profile is assumed in our approach: by finding an explicit solution to the dilaton equation of motion with realistic field configurations of Type IIB superstring on $A d S_{5} \otimes S^{5}$. While a spacelike singularity exists in the bulk in the dilaton profile proposed earlier [73], there is no singularity in the bulk even at the time of bounce since the CSTB model has a non-zero minimal radius at the bounce point. Furthermore we take the four dimensional bounce universe to be a closed FLRW without violating NEC. In [73] their result does not depend on the details of the time dependence of the boundary coupling. As this is all about the evolution of boundary fluctuations through a potential singularity in the bulk spacetime, we are not sure whether their result is applicable to general backgrounds and differently assumed dilaton profiles.

A clarifying remark on two different kinds of $k$-modes arisen in the study is perhaps warranted. In many of the early universe models, the Hubble parameter is no longer a constant. Different $k$-modes exit and reenter the Hubble horizon at different times: the mode numbers pick up an implicit time dependence as a result of the time dependence in the Hubble scale. This makes each $k$-mode in the primordial density perturbations pick up an implicit time dependence: only after this implicit time dependence is carefully taken into account can the time dependence in the spectrum be understood. This is thus the key to the stability analysis on the spectrum in the CSTB model [32,33].

The $k$-modes in (43) are, however, the $k$-modes of the gauge fields living on the boundary of the $A d S_{5}$. They are involved in the mapping procedure and merely encode the bulk dynamics holographically at particular points on the boundary. Therefore they cannot inject or remove any time dependence in the primordial spectrum. Once the dynamics are mapped onto the boundary, there is no more horizon crossing, the gauge fields evolve under their own equations of motion. And the information of the $k$ dependence in the power spectrum, which is generated during contraction phase of bulk, is fully encoded in the solutions of gauge field fluctuations on the boundary.
We have made several assumptions and approximations throughout the analysis. Different solutions of the dilaton could be obtained with different ansatz of the RamondRamond field configurations. We have simply chosen the most manageable, yet retaining the interesting physics, configuration. With higher orders of time dependence in the dilaton field we have to expand $M_{Y M}^{2}$ to the higher order in $t$ when solving (43). A systematic survey of the field configurations and their effects on the dilaton profile is beyond the scope of this paper. A dynamical mechanism, without prior assumptions on the parameters, to select out the correct values of the parameters is highly desirable. These are interesting physics, together with higher $\alpha$ / and higher string-loop effects on the string cosmological model, which we hope to address in future works.

Another line of research would be to properly set up and study the D-brane and anti-D-brane annihilation process for cosmological modelling. This constitutes the (quantum) basis for building early universe model in string theory. This allows us to go beyond the effective field theory approach and beyond kinematic analysis or symmetry arguments. A more realistic touch to string cosmology will eventually come from finding a consistent string compactification that yields a nonsingular universe along with testable predictions that stand up to the the array of precision cosmological observations.

Acknowledgements This research project has been supported in parts by the NSF China under Contract Nos. 11775110, 11690034 and 11405084. We also acknowledge the European Union's Horizon2020 research and innovation programme (RISE) under the Marie Skĺodowska-Curie Grant agreement No. 644121, and the Priority Academic Program Development for Jiangsu Higher Education Institutions (PAPD).

Open Access This article is distributed under the terms of the Creative Commons Attribution 4.0 International License (http://creativecomm ons.org/licenses/by/4.0/), which permits unrestricted use, distribution, and reproduction in any medium, provided you give appropriate credit to the original author(s) and the source, provide a link to the Creative Commons license, and indicate if changes were made.

Funded by SCOAP ${ }^{3}$.

\section{References}

1. D. Wands, Duality invariance of cosmological perturbation spectra. Phys. Rev. D 60, 023507 (1999). https://doi.org/10.1103/ PhysRevD.60.023507. arXiv:gr-qc/9809062

2. S. Gratton, J. Khoury, P.J. Steinhardt, N. Turok, Conditions for generating scale-invariant density perturbations. Phys. Rev. D 69, 103505 (2004). https://doi.org/10.1103/PhysRevD.69.103505. arXiv:astro-ph/0301395

3. S. Nojiri, S.D. Odintsov, V.K. Oikonomou, Modified gravity theories on a nutshell: inflation, bounce and late-time evolution. Phys. Rep. 692, 1 (2017). https://doi.org/10.1016/j.physrep.2017. 06.001. arXiv:1705.11098 [gr-qc]

4. R. Brandenberger, P. Peter, Bouncing cosmologies: progress and problems. Found. Phys. 47, 797 (2017). https://doi.org/10.1007/ s10701-016-0057-0. arXiv:1603.05834 [hep-th] 
5. D. Battefeld, P. Peter, A critical review of classical bouncing cosmologies. Phys. Rep. 571, 1 (2015). https://doi.org/10.1016/ j.physrep.2014.12.004. arXiv:1406.2790 [astro-ph.CO]

6. E. Wilson-Ewing, The matter bounce scenario in loop quantum cosmology. JCAP 1303, 026 (2013). https://doi.org/10.1088/ 1475-7516/2013/03/026. arXiv:1211.6269 [gr-qc]

7. M. Novello, S.E.P. Bergliaffa, Bouncing cosmologies. Phys. Rep. 463, 127 (2008). https://doi.org/10.1016/j.physrep.2008.04.006. arXiv:0802.1634 [astro-ph]

8. Y.F. Cai, D.A. Easson, R. Brandenberger, Towards a nonsingular bouncing cosmology. JCAP 1208, 020 (2012). https://doi.org/10. 1088/1475-7516/2012/08/020. arXiv:1206.2382 [hep-th]

9. Y.F. Cai, R. Brandenberger, X. Zhang, The matter bounce curvaton scenario. JCAP 1103, 003 (2011). https://doi.org/10.1088/ 1475-7516/2011/03/003. arXiv:1101.0822 [hep-th]

10. C. Lin, R.H. Brandenberger, L. Perreault Levasseur, A matter bounce by means of ghost condensation. JCAP 1104, 019 (2011). https://doi.org/10.1088/1475-7516/2011/04/ 019. arXiv:1007.2654 [hep-th]

11. A. Wang, Y. Wu, Thermodynamics and classification of cosmological models in the Horava-Lifshitz theory of gravity. JCAP 0907, 012 (2009). https://doi.org/10.1088/1475-7516/2009/07/ 012. arXiv:0905.4117 [hep-th]

12. Y.S. Piao, Proliferation in cycle. Phys. Lett. B 677, 1 (2009). https:// doi.org/10.1016/j.physletb.2009.05.009. arXiv:0901.2644 [gr-qc]

13. P. Peter, N. Pinto-Neto, Cosmology without inflation. Phys. Rev. D 78, 063506 (2008). https://doi.org/10.1103/PhysRevD.78.063506. arXiv:0809.2022 [gr-qc]

14. L.E. Allen, D. Wands, Cosmological perturbations through a simple bounce. Phys. Rev. D 70, 063515 (2004). https://doi.org/10.1103/ PhysRevD.70.063515. arXiv:astro-ph/0404441

15. J.E. Lidsey, D. Wands, E.J. Copeland, Phys. Rep. 337, 343 (2000). https://doi.org/10.1016/S0370-1573(00)00064-8. arXiv:hep-th/9909061

16. M. Gasperini, G. Veneziano, Pre-Big Bang in string cosmology. Astropart. Phys. 1, 317 (1993). https://doi.org/10.1016/ 0927-6505(93)90017-8. arXiv:hep-th/9211021

17. A. Buonanno, K.A. Meissner, C. Ungarelli, G. Veneziano, Quantum inhomogeneities in string cosmology. JHEP 9801, 004 (1998). https://doi.org/10.1088/1126-6708/1998/01/004. arXiv:hep-th/9710188

18. J. Khoury, B.A. Ovrut, P.J. Steinhardt, N. Turok, The ekpyrotic universe: colliding branes and the origin of the hot Big Bang. Phys. Rev. D 64, 123522 (2001). https://doi.org/10.1103/PhysRevD.64. 123522. arXiv:hep-th/0103239

19. D.H. Lyth, The Primordial curvature perturbation in the ekpyrotic universe. Phys. Lett. B 524, 1 (2002). https://doi.org/10.1016/ S0370-2693(01)01374-0. arXiv:hep-ph/0106153

20. F. Finelli, R. Brandenberger, On the generation of a scale invariant spectrum of adiabatic fluctuations in cosmological models with a contracting phase. Phys. Rev. D 65, 103522 (2002). https://doi.org/ 10.1103/PhysRevD.65.103522. arXiv:hep-th/0112249

21. Y.F. Cai, A. Marciano, D.G. Wang, E. Wilson-Ewing, Bouncing cosmologies with dark matter and dark energy. Universe 3(1), 1 (2016). https://doi.org/10.3390/universe3010001. arXiv:1610.00938 [astro-ph.CO]

22. A. Addazi, S. Alexander, Y.F. Cai, A. Marciano, Dark matter and baryogenesis in the Fermi-bounce curvaton mechanism. Chin. Phys. C 42(6), 065101 (2018). https://doi.org/10.1088/1674-1137/ 42/6/065101. arXiv:1612.00632 [gr-qc]

23. A. Sen, Tachyon matter. JHEP 0207, 065 (2002). https://doi.org/ 10.1088/1126-6708/2002/07/065. arXiv:hep-th/0203265

24. A. Sen, Rolling tachyon. JHEP 0204, 048 (2002). https://doi.org/ 10.1088/1126-6708/2002/04/048. arXiv:hep-th/0203211
25. G.W. Gibbons, Cosmological evolution of the rolling tachyon. Phys. Lett. B 537, 1 (2002). https://doi.org/10.1016/ S0370-2693(02)01881-6. arXiv:hep-th/0204008

26. A.H. Guth, D.I. Kaiser, Y. Nomura, Inflationary paradigm after Planck 2013. Phys. Lett. B 733, 112 (2014). https://doi.org/10. 1016/j.physletb.2014.03.020. arXiv:1312.7619 [astro-ph.CO]

27. A. Linde, Inflationary cosmology after planck, in Post-Planck Cosmology: Lecture Notes of the Les Houches Summer School: Volume 100, ed. by C. Deffayet, P. Peter, B. Wandelt, M. Zaldarriaga, L.F. Cugliandolo. https://doi.org/10.1093/acprof:oso/9780198728856. 003.0006

28. G. Hinshaw, [WMAP Collaboration], Nine-year wilkinson microwave anisotropy probe (WMAP) observations: cosmological parameter results. Astrophys. J. Suppl. 208, 19 (2013). https:// doi.org/10.1088/0067-0049/208/2/19. arXiv:1212.5226 [astroph.CO]

29. Planck Collaboration, Planck 2015 results. XVI. Isotropy and statistics of the CMB. Astron. Astrophys. 594, A16 (2016). https:// doi.org/10.1051/0004-6361/201526681. arXiv:1506.07135 [astro-ph.CO]

30. C. Li, L. Wang, Y.K.E. Cheung, Bound to bounce: a coupled scalar tachyon model for a smoothly bouncing or cyclic universe. Phys. Dark Univ. 3, 18 (2014). https://doi.org/10.1016/j.dark.2014.02. 001. arXiv:1101.0202 [gr-qc]

31. Y.K.E. Cheung, X. Song, S. Li, Y. Li, Y. Zhu, The CST bounce universe model A parametric study. Sci. China Phys. Mech. Astron. 62(1), 10011 (2019). https://doi.org/10.1007/s11433-018-9251-0. arXiv: 1601.03807 [gr-qc]

32. C. Li, Y.K.E. Cheung, Dualities between scale invariant and magnitude invariant perturbation spectra in inflationary/bouncing cosmos (2012). arXiv: 1211.1610 [gr-qc]

33. C. Li, Y.K.E. Cheung, The scale invariant power spectrum of the primordial curvature perturbations from the coupled scalar tachyon bounce cosmos. JCAP 1407, 008 (2014). https://doi.org/10.1088/ 1475-7516/2014/07/008. arXiv:1401.0094 [gr-qc]

34. C. Li, R.H. Brandenberger, Y.K.E. Cheung, Big Bounce genesis. Phys. Rev. D 90(12), 123535 (2014). https://doi.org/10.1103/ PhysRevD.90.123535. arXiv:1403.5625 [gr-qc]

35. Y.K.E. Cheung, J.U. Kang, C. Li, Dark matter in a bouncing universe. JCAP 1411(11), 001 (2014). https://doi.org/10.1088/ 1475-7516/2014/11/001. arXiv:1408.4387 [astro-ph.CO]

36. Y.K.E. Cheung, C. Li, J.D. Vergados, Big Bounce genesis and possible experimental tests: a brief review. Symmetry 8(11), 136 (2016). https://doi.org/10.3390/sym8110136. arXiv:1611.04027 [astro-ph.CO]

37. Y.K.E. Cheung, J.D. Vergados, Direct dark matter searches: test of the Big Bounce cosmology. JCAP 1502(02), 014 (2015). https:// doi.org/10.1088/1475-7516/2015/02/014. arXiv:1410.5710 [hep$\mathrm{ph}]$

38. J.D. Vergados, C.C. Moustakidis, Y.K.E. Cheung, H. Ejri, Y. Kim, Y. Lie, Light WIMP searches involving electron scattering. Adv. High Energy Phys. 2018, 6257198 (2018). https://doi.org/10.1155/ 2018/6257198. arXiv:1605.05413 [hep-ph]

39. Y.F. Cai, Exploring bouncing cosmologies with cosmological surveys. Sci. China Phys. Mech. Astron. 57, 1414 (2014). https://doi. org/10.1007/s11433-014-5512-3. arXiv:1405.1369 [hep-th]

40. A. Borde, A. Vilenkin, Singularities in inflationary cosmology: a review. Int. J. Mod. Phys. D 5, 813 (1996). https://doi.org/10.1142/ S0218271896000497. arXiv:gr-qc/9612036

41. A. Borde, A. Vilenkin, Eternal inflation and the initial singularity. Phys. Rev. Lett. 72, 3305 (1994). https://doi.org/10.1103/ PhysRevLett.72.3305. arXiv:gr-qc/9312022

42. L. Randall, R. Sundrum, An alternative to compactification. Phys. Rev. Lett. 83, 4690 (1999). https://doi.org/10.1103/PhysRevLett. 83.4690. arXiv:hep-th/9906064 
43. L. Randall, R. Sundrum, A Large mass hierarchy from a small extra dimension. Phys. Rev. Lett. 83, 3370 (1999). https://doi.org/ 10.1103/PhysRevLett.83.3370. arXiv:hep-ph/9905221

44. J.M. Maldacena, The large $\mathrm{N}$ limit of superconformal field theories and supergravity. Adv. Theor. Math. Phys. 2, 231 (1998). https:// doi.org/10.4310/ATMP.1998.v2.n2.a1. arXiv:hep-th/9711200

45. S.P. Kumar, V. Vaganov, Quasinormal modes and holographic correlators in a crunching AdS geometry. JHEP 1602, 065 (2016). https://doi.org/10.1007/JHEP02(2016)065. arXiv: 1512.07184 [hep-th]

46. A. Bzowski, T. Hertog, M. Schillo, Cosmological singularities encoded in IR boundary correlations. JHEP 1605, 168 (2016). https://doi.org/10.1007/JHEP05(2016)168. arXiv:1512.05761 [hep-th]

47. S.P. Kumar, V. Vaganov, Probing crunching AdS cosmologies. High Energ. Phys. 2016, 26 (2016). https://doi.org/10.1007/ JHEP02(2016)026. arXiv:1510.03281 [hep-th]

48. J.L.F. Barbon, E. Rabinovici, Holographic complexity and cosmological singularities. JHEP 1601, 084 (2016). https://doi.org/10. 1007/JHEP01(2016)084. arXiv:1509.09291 [hep-th]

49. N. Engelhardt, G.T. Horowitz, Holographic consequences of a no transmission principle. Phys. Rev. D 93(2), 026005 (2016). https://doi.org/10.1103/PhysRevD.93.026005. arXiv:1509.07509 [hep-th]

50. B. Heidenreich, M. Reece, T. Rudelius, Weak gravity strongly constrains large-field axion inflation. JHEP 1512, 108 (2015). https:// doi.org/10.1007/JHEP12(2015)108. arXiv:1506.03447 [hep-th]

51. N. Engelhardt, T. Hertog, G.T. Horowitz, Further holographic investigations of Big Bang singularities. JHEP 1507, 044 (2015). https://doi.org/10.1007/JHEP07(2015)044. arXiv:1503.08838 [hep-th]

52. A. Enciso, N. Kamran, Determining an asymptotically AdS Einstein spacetime from data on its conformal boundary. Gen. Relativ. Gravit. 47(12), 147 (2015). https://doi.org/10.1007/ s10714-015-1974-5. arXiv:1502.01622 [gr-qc]

53. S. Banerjee, S. Bhowmick, S. Chatterjee, S. Mukherji, A note on AdS cosmology and gauge theory correlator. JHEP 1506, 043 (2015). https://doi.org/10.1007/JHEP06(2015)043. arXiv:1501.06317 [hep-th]

54. L. Battarra, M. Koehn, J.L. Lehners, B.A. Ovrut, Cosmological perturbations through a non-singular ghost-condensate/Galileon bounce. JCAP 1407, 007 (2014). https://doi.org/10.1088/ 1475-7516/2014/07/007. arXiv:1404.5067 [hep-th]

55. N. Engelhardt, T. Hertog, G.T. Horowitz, Holographic signatures of cosmological singularities. Phys. Rev. Lett. 113, 121602 (2014). https://doi.org/10.1103/PhysRevLett.113.121602. arXiv: 1404.2309 [hep-th]

56. I.A. Morrison, Boundary-to-bulk maps for AdS causal wedges and the Reeh-Schlieder property in holography. JHEP 1405, 053 (2014). https://doi.org/10.1007/JHEP05(2014)053. arXiv:1403.3426 [hep-th]

57. R.H. Brandenberger, C. Kounnas, H. Partouche, S.P. Patil, N. Toumbas, Cosmological perturbations across an S-brane. JCAP 1403, 015 (2014). https://doi.org/10.1088/1475-7516/2014/03/ 015. arXiv:1312.2524 [hep-th]

58. A. Enciso, N. Kamran, A singular initial-boundary value problem for nonlinear wave equations and holography in asymptotically anti-de Sitter spaces (2014). arXiv:1310.0158 [math.AP]

59. M. Smolkin, N. Turok, Dual description of a 4d cosmology (2012). arXiv:1211.1322 [hep-th]

60. A. Enciso, N. Kamran, Causality and the conformal boundary of AdS in real-time holography. Phys. Rev. D 85, 106016 (2012). https://doi.org/10.1103/PhysRevD.85.106016. arXiv:1203.2743 [math-ph]
61. J.L.F. Barbon, E. Rabinovici, AdS crunches. CFT falls and cosmological complementarity. JHEP 1104, 044 (2011). https://doi.org/ 10.1007/JHEP04(2011)044. arXiv:1102.3015 [hep-th]

62. A. Awad, S.R. Das, A. Ghosh, J.H. Oh, S.P. Trivedi, Slowly varying dilaton cosmologies and their field theory duals. Phys. Rev. D 80, 126011 (2009). https://doi.org/10.1103/PhysRevD.80.126011. arXiv:0906.3275 [hep-th]

63. A. Awad, S.R. Das, S. Nampuri, K. Narayan, S.P. Trivedi, Gauge theories with time dependent couplings and their cosmological duals. Phys. Rev. D 79, 046004 (2009). https://doi.org/10.1103/ PhysRevD.79.046004. arXiv:0807.1517 [hep-th]

64. B. Craps, F. De Roo, O. Evnin, Quantum evolution across singularities: the case of geometrical resolutions. JHEP 0804, 036 (2008). https://doi.org/10.1088/1126-6708/2008/04/036. arXiv:0801.4536 [hep-th]

65. A. Awad, S.R. Das, K. Narayan, S.P. Trivedi, Gauge theory duals of cosmological backgrounds and their energy momentum tensors. Phys. Rev. D 77, 046008 (2008). https://doi.org/10.1103/ PhysRevD.77.046008. arXiv:0711.2994 [hep-th]

66. N. Turok, B. Craps, T. Hertog, From big crunch to Big Bang with AdS/CFT. arXiv:0711.1824 [hep-th]

67. C.S. Chu, P.M. Ho, Time-dependent AdS/CFT duality. II. Holographic reconstruction of bulk metric and possible resolution of singularity. JHEP 0802, 058 (2008). https://doi.org/10.1088/ 1126-6708/2008/02/058. arXiv:0710.2640 [hep-th]

68. S.R. Das, J. Michelson, K. Narayan, S.P. Trivedi, Time dependent cosmologies and their duals. Phys. Rev. D 74, 026002 (2006). https://doi.org/10.1103/PhysRevD.74.026002. arXiv:hep-th/0602107

69. C.S. Chu, P.M. Ho, Time-dependent AdS/CFT duality and null singularity. JHEP 0604, 013 (2006). https://doi.org/10.1088/ 1126-6708/2006/04/013. arXiv:hep-th/0602054

70. A. Hamilton, D.N. Kabat, G. Lifschytz, D.A. Lowe, Local bulk operators in AdS/CFT: a boundary view of horizons and locality. Phys. Rev. D 73, 086003 (2006). https://doi.org/10.1103/ PhysRevD.73.086003. arXiv:hep-th/0506118

71. T. Hertog, G.T. Horowitz, Holographic description of AdS cosmologies. JHEP 0504, 005 (2005). https://doi.org/10.1088/ 1126-6708/2005/04/005. arXiv:hep-th/0503071

72. R. Durrer, F. Vernizzi, Adiabatic perturbations in pre-Big Bang models: matching conditions and scale invariance. Phys. Rev. D 66, 083503 (2002). https://doi.org/10.1103/PhysRevD.66.083503. arXiv:hep-ph/0203275

73. R.H. Brandenberger, E.G.M. Ferreira, I.A. Morrison, Y.F. Cai, S.R. Das, Y. Wang, Fluctuations in a cosmology with a spacelike singularity and their gauge theory dual description. Phys. Rev. D 94(8), 083508 (2016). https://doi.org/10.1103/PhysRevD. 94.083508. arXiv:1601.00231 [hep-th]

74. E.G.M. Ferreira, R. Brandenberger, Holographic curvature perturbations in a cosmology with a space-like singularity. JCAP 1607, 030 (2016). https://doi.org/10.1088/1475-7516/2016/07/ 030. arXiv:1602.08152 [hep-th]

75. N.S. Deger, Time-dependent AdS backgrounds from S-Branes. Phys. Lett. B 762, 209 (2016). https://doi.org/10.1016/j.physletb. 2016.07.024. arXiv:1606.00674 [hep-th]

76. S.R. Das, Old and new scaling laws in quantum quench. PTEP 2016(12), 12C107 (2016). https://doi.org/10.1093/ptep/ptw146. arXiv:1608.04407 [hep-th]

77. S. Chatterjee, S.P. Chowdhury, S. Mukherji, Y.K. Srivastava, Nonvacuum AdS cosmology and comments on gauge theory correlator. Phys. Rev. D 95(4), 046011 (2017). https://doi.org/10.1103/ PhysRevD.95.046011. arXiv:1608.08401 [hep-th]

78. E. Bergshoeff, R. Kallosh, T. Ortin, D. Roest, A. Van Proeyen, New formulations of $\mathrm{D}=10$ supersymmetry and $\mathrm{D} 8-\mathrm{O} 8$ domain walls. Class. Quantum Gravity 18, 3359 (2001). https://doi.org/10.1088/ 0264-9381/18/17/303. arXiv:hep-th/0103233 
79. J. Polchinski, String theory, vol. 2: Superstring theory and beyond (1998). https://doi.org/10.1017/CBO9780511618123

80. K. Sfetsos, D.C. Thompson, On non-abelian T-dual geometries with Ramond fluxes. Nucl. Phys. B 846, 21 (2011). https://doi.org/ 10.1016/j.nuclphysb.2010.12.013. arXiv:1012.1320 [hep-th]
81. N.T. Macpherson, C. Núñez, L.A. Pando Zayas, V.G.J. Rodgers, C.A. Whiting, Type IIB supergravity solutions with $\mathrm{AdS}_{5}$ from Abelian and non-Abelian $\mathrm{T}$ dualities. JHEP 1502, 040 (2015). https://doi.org/10.1007/JHEP02(2015)040. arXiv:1410.2650 [hep-th] 